\title{
Correction to: Salvage fractionated stereotactic re-irradiation (FSRT) for patients with recurrent high grade gliomas progressed after bevacizumab treatment
}

\author{
Wenyin Shi ${ }^{1}$ - Erik S. Blomain ${ }^{2} \cdot$ Joshua Siglin $^{3} \cdot$ Joshua D. Palmer $^{4} \cdot$ Tu Dan $^{5} \cdot$ Yang Wang $^{6} \cdot$ Maria Werner-Wasik $^{1}$. \\ Jon Glass ${ }^{7}$. Lyndon $\mathrm{Kim}^{7}$. Voichita Bar $\mathrm{Ad}^{1}$. Deepak Bhamidipati ${ }^{8}$. James J. Evans ${ }^{7} \cdot$ Kevin Judy $^{7}$. \\ Christopher J. Farrell ${ }^{7}$. David W. Andrews ${ }^{7}$
}

Published online: 8 January 2018

(c) Springer Science+Business Media, LLC, part of Springer Nature 2018

\section{Correction to: Journal of Neuro-Oncology https://doi.org/10.1007/s11060-017-2709-0}

The fourth author's name was incorrect in the initial online publication. The original article has been corrected.

The original article can be found online at https://doi.org/10.1007/ s11060-017-2709-0.

Wenyin Shi

Wenyin.Shi@jefferson.edu

1 Department of Radiation Oncology, Sidney Kimmel Medical College, Thomas Jefferson University, Philadelphia, PA, USA

2 Jefferson College of Population Health, Thomas Jefferson University, Philadelphia, PA, USA

3 Department of Radiation Oncology, University of Pittsburgh Medical Center, Altoona Cancer Center, Altoona, PA, USA

4 Department of Radiation Oncology, Ohio State University, Columbus, OH, USA

5 Department of Radiation Oncology, University of Texas Southwestern, Dallas, TX, USA

6 Cyberknife Center, Huashan Hospital Pudong, Fudan University, Shanghai, China

7 Department of Neurological Surgery, Thomas Jefferson University, Philadelphia, PA, USA

8 Sidney Kimmel Medical College, Thomas Jefferson University, Philadelphia, PA, USA 